\title{
The lateralized readiness potential as an on-line measure of central response activation processes
}

\author{
MARTIN EIMER \\ University of Cambridge, Cambridge, England
}

\begin{abstract}
The lateralized readiness potential (LRP) is an electrophysiological indicator of the central activation of motor responses. Procedures for deriving the LRP on the basis of event-related brain potential (ERP) waveforms obtained over the left and right motor cortices are described, and some findings are summarized that show that the LRP is likely to reflect activation processes within the motor cortex. Two experiments investigating spatial S-R compatibility effects are reported that demonstrate that, because of systematic overlaps of motor and nonmotor asymmetries, LRP waveforms derived by the double subtraction method cannot always be interpreted unequivocally in terms of response activation. Such confounds can be detected when LRP waveforms are compared with difference waveforms obtained by the double subtraction method from ERPs elicited at other lateral scalp sites.
\end{abstract}

One general and widely used approach in the experimental study of cognitive processes is that of mental chronometry. The term mental chronometry, as introduced by Posner (1978), refers to investigations of the structure and time course of human information processing. Discriminative and goal-directed performance is assumed to be the result of an interplay of different cognitive operations that are organized within processing stages-some primarily dealing with perceptual analysis, classification, and identification; others with response selection, preparation, and execution; and still others with control functions or attentional selectivity. The aim of the chronometrist is to identify these cognitive operations, describe their interactions and their temporal characteristics, and specify how their coordinated activity enables the organism to perform adaptively in its environment.

Most studies in the field of mental chronometry have employed performance measures (such as response speed or accuracy) as dependent variables. A potential problem with this approach is that covert cognitive processes are studied on the basis of overt behavior that is usually executed after these processes have been terminated. Interpreting overt performance as evidence for or against hypotheses about the properties of covert cognitive processes thus inevitably requires inferential processes (see Coles, 1989). Such inferences are often based on rather general assumptions about the organization of cognitive operations (e.g., on discrete serial stage models of information

The present research was supported by the Max Planck Institute for Psychological Research and by Grant Ei 266/4-1 from the Deutsche Forschungsgemeinschaft. The author thanks Erich Schröger (who served as action editor for this article), Werner Sommer, and Rolf Verleger for valuable comments. Correspondence concerning this article should be addressed to M. Eimer, Department of Experimental Psychology, University of Cambridge, Downing St., Cambridge, CB2 3EB, UK (e-mail: me209@cam.ac.uk). processing). Whenever such assumptions are called into question, specific inferences from behavioral performance measures to cognitive processes are also challenged. A more direct access to cognitive activity is therefore obviously desirable. Event-related brain potential (ERP) measures provide such an access. In contrast to behavioral measures, ERPs can be recorded on-line and continuously while cognitive processes are operating and thus can, in principle, provide fine-grained temporal information about the functional organization of information processing. In order to infer, on the basis of ERP recordings, the structure and time course of cognitive processes, ERP components have to be related to specific cognitive operations (for a discussion of the concept of ERP components, see van Boxtel, 1998). One major aim in ERP research is to describe relationships between ERP components and specific cognitive processes, such as perceptual analysis, stimulus discrimination, classification or identification, memory operations, or response selection and activation (see Coles, Gratton, \& Fabiani, 1990, for a further discussion of this issue). When the functional significance of an ERP component is known, it can serve as a useful tool for mental chronometry.

An ERP component that has gained considerable interest in the last decade, both from psychophysiologists and from cognitive psychologists, is the lateralized readiness potential (LRP). The LRP is assumed to be related to selective response activation processes. In the first part of this paper, the procedure for deriving the LRP on the basis of ERP waveforms obtained from electrodes located over left and right motor areas of the brain is described, and some general methodological issues (appropriate recording sites, the minimum number of single sweeps needed to compute reliable LRP waveforms, stimulus- and response-locked averaging) will be briefly discussed. The second part presents findings showing that the LRP may be interpreted as an index for selective 
central response activation processes, although it is not yet entirely clear which aspects of central response activation are reflected by the LRP. In the third part, a specific methodological problem for the application of the LRP in mental chronometry will be discussed: Under certain experimental conditions, LRP deflections do not uniquely reflect central response activation processes, because they will be systematically affected by lateralized activity that originates from nonmotor areas of the brain. Overlaps between motor and nonmotor activity in the LRP may be detected when LRP waveforms are compared with measures of lateralized brain activity obtained from different scalp sites. This will be illustrated by two empirical examples. When applied properly, the LRP method will be an important tool for mental chronometry.

\section{The Lateralized Readiness Potential: History and Derivation}

The LRP was introduced into the experimental literature in 1988 simultaneously by research groups in Groningen (De Jong, Wierda, Mulder, \& Mulder, 1988) and Illinois (Gratton, Coles, Sirevaag, Eriksen, \& Donchin, 1988). It was initially termed corrected motor asymmetry (CMA) by the former group, but the term lateralized readiness potential (LRP), introduced by Gratton et al. (1988), is now generally used. The LRP is derived from scalp potentials that precede limb movements. Such potentials were first described by Kornhuber and Deecke (1965), who found a gradually increasing negative shift prior to the initiation of a hand movement, starting about 1 sec before movement onset. This so-called Bereitschaftspotential (readiness potential, RP) initially is equally large over both hemispheres but begins to lateralize before the onset of the response, with larger amplitudes found over the hemisphere contralateral to the response side. This lateralization is maximal for recording sites above the motor cortex. Kutas and Donchin (1980) observed that the beginning of the lateralization of the $\mathrm{RP}$ reflects the point in time at which the response side (left hand vs. right hand) is determined. They argued that the lateralization of the RP indicates the differential engagement of the left and right motor cortices in the preparation and initiation of unimanual motor responses.

A similar negative-going potential has also been observed in warned reaction time tasks in the interval between the warning stimulus and the imperative stimulus (Walter, Cooper, Aldridge, McCallum, \& Winter, 1964). This potential has been termed contingent negative variation (CNV). When the warning stimulus specifies the hand that has to be used in response to the imperative stimulus, the later part of the CNV becomes asymmetric, with larger amplitudes over the hemisphere contralateral to the response hand (Syndulko \& Lindsley, 1977). It is not clear whether the later phases of the RP and the CNV reflect equivalent or functionally different processes (see Rohrbaugh \& Gaillard, 1983; van Boxtel, 1994). Experiments that measured the LRP as a dependent variable have employed $\mathrm{S} 1-\mathrm{S} 2$ paradigms (in which a $\mathrm{CNV}$ is as- sumed to be elicited) as well as paradigms that give rise to RPs. Thus, a number of studies have measured LRPs that might be described more strictly as lateralized CNV waveforms.

The LRP is computed on the basis of ERP potentials recorded prior to and during the execution of a response over the left and right motor cortices. The exact locations of the recordings sites vary slightly between experimental studies. Frequently chosen recording site pairs are $\mathrm{C}^{\prime}$ and $\mathrm{C4}^{\prime}$, located $1 \mathrm{~cm}$ anterior of the $\mathrm{C} 3$ and $\mathrm{C} 4$ sites specified by the 10-20 system (Jasper, 1958). Miller and Hackley (1992) recorded the LRP from electrodes located $1 \mathrm{~cm}$ anterior and superior to $\mathrm{C} 3$ and C4. Gratton et al. (1988) employed electrodes situated $4 \mathrm{~cm}$ to the left and right of $\mathrm{Cz}$, whereas Smulders, Kok, Kenemans, and Bashore (1995) recorded directly from C3 and C4. Sommer, Leuthold, and Ulrich (1994) showed that the amplitude and overall shape of the LRP is relatively constant at these locations but that LRP amplitude gets considerably smaller for slightly more anterior and superior positions.

The method for deriving the LRP is illustrated in Figure 1 on the basis of grand-averaged ERP waveforms obtained from 10 subjects who were instructed to respond with a left-hand buttonpress whenever the letter $W$ was presented at the center of a computer screen and with a right-hand response whenever the letter $M$ was presented. A cue stimulus that indicated with $75 \%$ validity the identity of the target letter was delivered $900 \mathrm{msec}$ before letter onset. LRP waveforms that were obtained in the cue-target interval of this experiment have already been reported by Eimer, Hommel, and Prinz (1995, Experiment 4 ). ${ }^{1}$ Figure 1 (top row) shows the ERP waveforms elicited in response to the two target letters over the left $\left(\mathrm{C}^{\prime}\right)$ and right $\left(\mathrm{C}^{\prime}\right)$ motor cortex. Following the target mapped to a left-hand response, the ERP obtained over the right motor cortex $\left(\mathrm{C} 4^{\prime}\right)$ starts to become more negative than the ERP waveform obtained at $\mathrm{C} 3^{\prime}$. The opposite pattern can be seen for targets requiring a righthand response. Strictly speaking, all ERPs displayed in Figure 1 (top row) show a marked positive-going shift in response to the target stimuli, but this positivity gradually becomes larger over the hemisphere ipsilateral to the required response. The computation of an LRP does not presuppose that clearly detectable negative-going potentials like the RP or the CNV are present in the ERP waveforms obtained over the left and right motor cortex.

These lateralized negativities are made explicit in the LRP waveforms with the help of the double subtraction method. As a first step, $\mathrm{C}^{\prime}-\mathrm{C}^{\prime}$ difference amplitudes are computed separately for trials in which stimuli assigned to left-hand responses and stimuli assigned to right-hand responses are presented (Figure 1, middle row). The resulting difference waveform for right-hand responses is then subtracted from the difference waveform for left-hand responses, which results in the LRP waveform (Figure 1, bottom row). As a result of these two subtractions, lateralized negativities recorded over the 


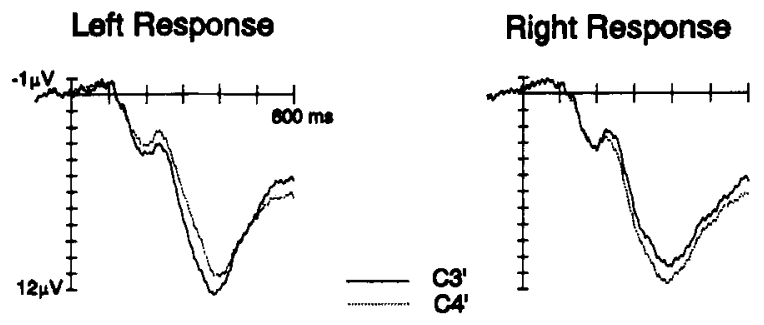

Subtraction 1: $\mathrm{C3}^{\prime} \cdot \mathrm{CA}^{\prime}$

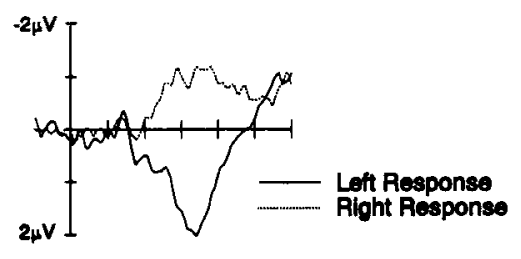

Subtraction 2: $\left(C 3^{\prime}-C^{\prime}\right)(L)-\left(C 3^{\prime}-C 4^{\prime}\right)(R)$

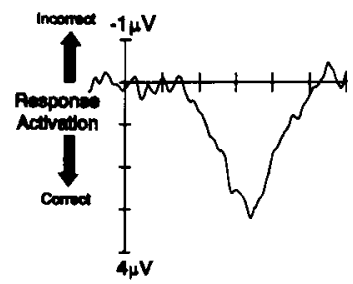

Figure 1. Computation of the lateralized readiness potential (LRP) with the double subtraction method on the basis of event-related brain potential (ERP) waveforms elicited at electrodes $\mathrm{C3}^{\prime}$ (left hemisphere) and $\mathrm{C4}^{\prime}$ (right hemisphere). Top panels: Grand-averaged ERP waveforms from 10 subjects elicited at $\mathrm{C3}^{\prime}$ (solid lines) and $\mathrm{C4}^{\prime}$ (dashed lines) in response to stimuli requiring a left-hand response (left side) and to stimuli requiring a right-hand response (right side). Middle panel: Difference waveforms resulting from subtracting the ERPs obtained at C4' from the ERPs obtained at $\mathrm{C3}^{\prime}$ 'separately for left-hand responses (solid line) and right-hand responses (dashed line). Bottom panel: LRP waveform resulting from subtracting the $\mathrm{C3}^{\prime}-\mathrm{C4}^{\prime}$ difference waveform for right-hand responses from the $\mathrm{C3}^{\prime}-\mathrm{C4}^{\prime}$ difference waveform for left-hand responses. A downwardgoing (positive) deflection indicates an activation of the correct response; an upward-going (negative) deflection indicates an activation of the incorrect response.

motor cortex contralateral to the to-be-executed response are reflected in positive (downward-going) deflections in the LRP waveforms. Larger negativities recorded over the motor cortex ipsilateral to the required response (indicating an activation of the incorrect response) are reflected in negative (upward-going) deflections in the LRP waveforms. With the double subtraction method, lateralized ERP activity that is unrelated to the side of the required response will be canceled out. For example, Figure 1 (top row) shows that between 500 and $600 \mathrm{msec}$ after stimulus onset, the ERPs recorded over the left motor cortex $\left(\mathrm{C}^{\prime}\right)$ tend to be less positive than the ERPs recorded over $\mathrm{C}^{\prime}$. However, because this asymmetry is present for left-hand responses as well as for right-hand responses, it is eliminated by the second subtraction. The LRP waveforms derived by the double subtraction method will only deviate from zero when lateralized ERP activity varies with the side of the to-beexecuted movement. It is therefore often assumed that LRP waveforms exclusively reflect movement-related ERP activity. As will be discussed below, this assumption may not always be warranted.

The double subtraction method, as illustrated in Figure 1, was introduced by De Jong et al. (1988). It should be noted that slightly different methods for deriving the LRP have also been employed. For example, Coles (1989) described an alternative way of computing LRP waveforms (the averaging method). Here, ERP waveforms recorded over motor areas ipsilateral to the responding hand are subtracted from ERP waveforms obtained over the contralateral motor cortex. These subtractions are performed separately for left-hand and right-hand responses. The resulting two difference waveforms are averaged, and the averaged waveform is the LRP. In principle, the procedures described by De Jong et al. (1988) and Coles are equivalent. There are, however, two differences with respect to the resulting LRP waveforms. With the averaging method, an activation of the correct response is reflected in the LRP by negative amplitude values, whereas incorrect response activation is reflected by positive LRP amplitudes. The double subtraction method yields opposite polarities. In addition to this sign reversal, the LRP amplitude values obtained with the double subtraction method are exactly twice as large as the values obtained with the averaging method described by Coles. These differences between the double subtraction and the averaging methods can be easily seen when the respective formulas for deriving the LRP are compared.

Double subtraction: $\left(\mathrm{C}^{\prime}-\mathrm{C}^{\prime}\right)_{\text {left hand }}-\left(\mathrm{C} 3^{\prime}-\mathrm{C}^{\prime}\right)_{\text {right hand }}$

$$
\text { Averaging: } \frac{\left(\mathrm{C} 4^{\prime}-\mathrm{C} 3^{\prime}\right)_{\text {left hand }}+\left(\mathrm{C} 3^{\prime}-\mathrm{C} 4^{\prime}\right)_{\text {right hand }}}{2}
$$

LRP waveforms reflect the lateralization of ERP activity observed prior to movement onset that is assumed to be related to a central activation of an unimanual response. It should be noted that LRPs can be measured not only immediately before and during the execution of left-hand and right-hand responses. LRPs have been obtained in the foreperiod of a warned RT task, following precues that predicted the likely side of the upcoming response (see Gehring, Gratton, Coles, \& Donchin, 1992), as well as under conditions in which one (irrelevant) stimulus attribute signaled a unimanual response, whereas another (relevant) attribute indicated that no response was to be executed at all and no overt response was elicited (see Miller \& Hackley, 1992). Because of its independence from the actual execution of an overt response, the LRP is often referred to as an index of partial response activation. 
Like any other ERP measure, the LRP is based on waveforms that are the result of averaging over a number of single trials. When compared to other ERP components, such as the P3, the LRP is quite small in amplitude. In order to obtain a reasonable signal-to-noise ratio, a relatively large number of single trials should be included in the averages. Although it is not possible to give general guidelines with respect to the minimum number of trials needed to compute reliable LRP waveforms, at least 40-50 trials should be included in the averaged ERP waveforms for both left-hand and right-hand responses. Since both response sides should contribute to the LRP waveforms with equal weight, special care must be taken that an approximately equal number of left-hand and right-hand response trials is included in the averages. This will not be the case in situations in which an unequal number of left-hand and right-hand response trials is delivered or in which overt response errors are unequally distributed between response hands.

The LRP can be computed on the basis of stimuluslocked averaged waveforms as well as on the basis of response-locked averages. In stimulus-locked LRPs, latency measures indicate the time between stimulus onset and specific LRP deflections (e.g., LRP onset). In response-locked LRPs, these latency measures refer to the distance in time between an LRP deflection and the overt response. Stimulus-locked LRP onset latency is determined by cognitive processes that occur prior to the selective activation of a response, whereas responselocked LRP onset latency reflects the duration of processes that take place between LRP onset and response execution. To find out whether experimental factors affect processes prior to or after the start of selective response activation, LRP onset latencies can be determined separately for stimulus-locked and response-locked LRPs (for applications of this procedure, see Leuthold, Sommer, \& Ulrich, 1996; Miller \& Ulrich, in press; Osman \& Moore, 1993).

\section{The LRP as an Index of Selective Central Response Activation}

The LRP has been employed as a dependent variable in numerous experimental investigations, including studies on stimulus-response compatibility (De Jong, Liang, \& Lauber, 1994; Eimer, 1995; Valle-Inclán, 1996), the partial transfer of stimulus information to motor stages (Miller \& Hackley, 1992; Osman, Bashore, Coles, Donchin, \& Meyer, 1992), response precuing (De Jong et al., 1988; Gehring et al., 1992; Leuthold et al., 1996), dualtask interference (Osman \& Moore, 1993), executive control processes (De Jong, Coles, Logan, \& Gratton, 1990), and sequence learning (Eimer, Goschke, Schlaghecken, \& Stürmer, 1996). In all of these studies, it is assumed that the LRP is a valid index for the central activation of unimanual responses. This section presents some findings that support this assumption.

Physiological studies have shown that the RP is generated in the primary motor cortex and in the supple- mentary motor areas of both hemispheres, and that this activity is larger in the hemisphere contralateral to the side of a to-be-executed movement, (cf. Arezzo \& Vaughan, 1975; Ikeda, Lüders, Burgess, \& Shibasaki, 1992; Okada, Williamson, \& Kaufman, 1982). For example, Gemba and Sasaki (1990) recorded in the monkey motor cortex surface-negative, depth-positive potentials prior to the onset of hand movements which indicates that the generator process is located in the motor cortex. In addition, RP amplitudes obtained for tongue, hand, and foot movements vary along the lateral central plane of the scalp, which corresponds closely to the anatomical localization of the respective motor projection areas (Vaughan, Costa, $\&$ Ritter, 1968). An initially surprising result has been reported by Brunia (1980), who found that foot movements were preceded by an enlarged negativity over ipsilateral motor areas. This has been explained by the anatomical localization of the primary motor area controlling the feet. This area is located medially within the longitudinal fissure in such a way that the current dipole that is generated when a unilateral foot movement is prepared points toward the ipsilateral scalp electrode, thus producing an ipsilaterally maximal RP (see also Böcker, Brunia, \& Cluitmans, 1994). Accordingly, Osman and Moore (1993) found that the LRP reverses polarity for foot as compared with hand responses. Because of the specific anatomical organization of the motor cortex, this polarity reversal can be seen as strong evidence that the LRP is indeed generated in the primary motor cortex.

In addition to such anatomical evidence, strong functional ties between the LRP and motor activation processes have also been reported. For example, the activity of many cells in the motor cortex closely parallels the onset and time course of the LRP (Requin, 1985). The existence of a fixed relationship between the LRP and the onset of a peripheral motor response has also been demonstrated by Gratton et al. (1988), who found that EMG activity began when the LRP reached a fixed threshold value, regardless of the actual response latency or accuracy.

These findings suggest that the processes responsible for the LRP are most likely located in the motor cortex and that the onset and time course of these processes is closely tied to the activation and execution of motor responses. It should be noted, however, that localizing the generator processes of the LRP within the primary motor cortex does not necessarily imply that the processes reflected by the LRP are exclusively motor in nature. Requin and Riehle (1995) measured the activity of single neurons in the monkey primary motor cortex and divided the neuron population into three types, depending on the temporal relation of the cells' activity to stimulus and movement onset. Although the majority of cells were classified as motor (with a pattern of activity time-locked to movement onset), a number of other cells were classified as sensory, since their activity was primarily timelocked to the onset of the stimulus. Although the functional status of these sensory neurons within the monkey 
primary motor cortex is still unclear, these findings may guard against a simple inference from the presence of brain activity within the motor cortex to the conclusion that this activity has to be functionally characterized as purely motor.

In addition, it is unclear which aspects of central response activation are reflected by the LRP. Only a few studies so far have investigated whether the LRP is systematically affected by response parameters such as direction, complexity, velocity, or force. Sommer et al. (1994) found that neither response force nor the rate of force production affected the LRP and concluded that the LRP merely reflects the relative activation of response alternatives but not a specific recruitment of force units. However, Leuthold et al. (1996) observed effects on the LRP recorded in the foreperiod of a cued choice reaction time task that seemed to indicate that the LRP does reflect the selection of specific muscle groups involved in a response-namely, that when a precue specified both movement direction (extension vs. flexion) and response hand, a larger foreperiod LRP was observed than was when the cue merely signaled the response hand.

\section{The Overlap of Motor and Nonmotor Activity in the LRP: The Case of Lateralized Stimuli}

Although the LRP may be interpreted as an index of central response activation and has been successfully applied in numerous experimental studies, there are some potential methodological problems that deserve consideration. One issue of particular importance for mental chronometry is the determination of the exact point in time at which sensory information starts to affect motor processing. If this is investigated with the help of the LRP, it is crucial to specify precisely the onset latencies of LRP waveforms. Since LRP waveforms are a result of averaging, the estimated onset latency may be affected disproportionately by a small subset of all trials. For stimulus-locked LRPs, it is likely that the onset is determined primarily by trials with short LRP onset latencies. For response-locked LRPs, onset estimates might be affected mostly by trials in which the interval between LRP onset and response execution is large. It is unclear which methods are best suited for determining LRP onset (for further discussions of this problem, see Schwarzenau, Falkenstein, Hoormann, \& Hohnsbein, 1998; Smulders, Kenemans, \& Kok, 1996). An interesting new method for computing LRP onset has recently been described by Miller, Patterson, and Ulrich (in press). The question as to how to determine onset latencies is, of course, not restricted to the LRP but is an important issue for ERP studies in general (see Gratton, Kramer, Coles, \& Donchin, 1989; van Boxtel, 1998).

Another potential methodological problem related to the LRP has to do with the fact that, under certain conditions, LRP waveforms do not exclusively reflect central response activation but are also systematically affected by brain activity presumably unrelated to motor pro- cesses. Because this overlap of motor and nonmotor activity is often not obvious at first glance and may thus give rise to functional interpretations that are clearly not warranted, the next sections will be devoted to a discussion of this problem. As described before, the double subtraction method is applied in deriving the LRP from averaged ERP waveforms because it is assumed that, with the help of this method, all activity not related to response-related processing is eliminated. However, there are experimental circumstances in which the LRP cannot be regarded as a valid index of partial response activation.

Such a situation can be encountered in studies of spatial stimulus response ( $S-R$ ) compatibility. Spatial $S-R$ compatibility effects can be observed when lateralized stimuli and responses are used. Responses are usually faster when stimuli and responses are located on the same side than when they are located on opposite sides, even when stimulus location is irrelevant for response selection. For example, when left-hand and right-hand responses are assigned to squares and circles, respectively, and these stimuli are presented randomly in the left or right visual field, RTs are faster in compatible trials in which stimulus and response locations correspond than in incompatible trials in which stimuli appear at the side contralateral to the required response (Wallace, 1971). This so-called Simon effect (Simon, 1969) has been explained by assuming that lateralized stimuli automatically activate spatially corresponding responses, which results in a correct response activation in compatible trials and in an activation of the incorrect response in incompatible trials (Kornblum, Hasbroucq, \& Osman, 1990; for an alternative view, see Hasbroucq \& Guiard, 1991). LRP measures seem to be ideally suited for testing this interpretation - that is, when horizontally lateralized stimuli and responses are employed, an early incorrect response activation should be observed for incompatible trials, whereas the correct response should be partially activated at the same time in compatible trials.

Figure 2 (bottom) shows the LRP waveforms for compatible and incompatible trials that were derived by the double subtraction method on the basis of ERPs from 10 subjects in an experiment on visual-spatial attention and spatial S-R compatibility that is described in detail in Eimer (1993, Experiment 1a). Subjects were instructed to respond with a left-hand buttonpress to the target letter $\mathrm{M}$ and with a right-hand buttonpress to the target letter W. These letters were presented randomly in the left and right visual field (with a horizontal distance of $6^{\circ}$ from the screen center). A precue that indicated with $75 \%$ validity the location of the upcoming target letter was presented $900 \mathrm{msec}$ before target onset. Only the data obtained in trials in which target location was correctly indicated by the cue are presented in Figures 2 and 3 . On compatible trials, the target letter was presented ipsilateral to the required response, whereas on incompatible trials, the required response was contralateral to the visual field where the target letter appeared (Figure 2, top row). A Simon effect was elicited under these condi- 


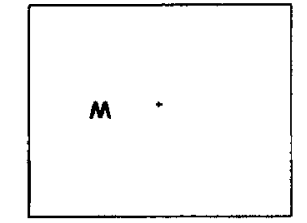

Left Response - Compatible

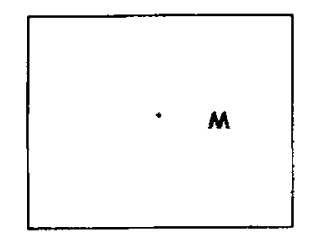

Left Response - Incompatible

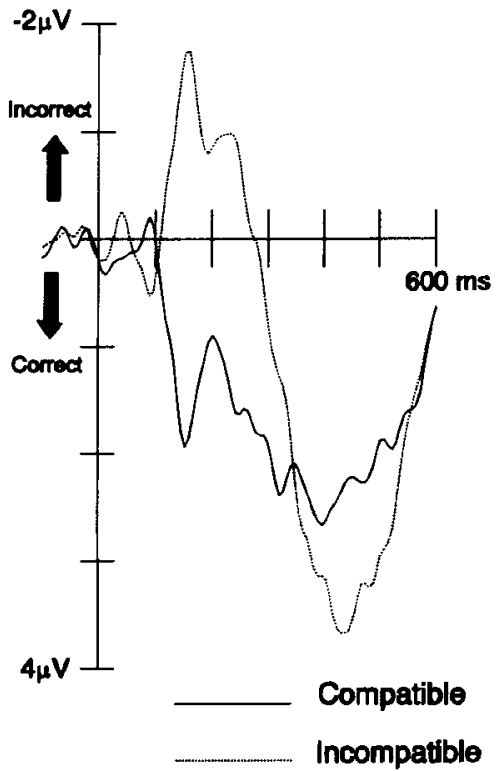

Figure 2. Top: Examples of stimulus displays in an experiment on spatial stimulus-response compatibility (Eimer, 1993, Experiment 1a) in which stimulus and response sides could either be compatible (left side) or incompatible (right side). Bottom: Grand-averaged LRP waveforms from 10 subjects, elicited in compatible trials (solid line) and in incompatible trials (dashed line).

tions, with mean RTs in compatible and incompatible trials of $427 \mathrm{msec}$ and $443 \mathrm{msec}$, respectively. The LRP waveforms, as shown in Figure 2 (bottom), revealed an early negative deflection (indicating an activation of the incorrect response) on incompatible trials and an early positive deflection (indicating correct response activation) on compatible trials.

Although these results seem to provide strong evidence in favor of the automatic response activation account, the LRP waveforms shown in Figure 2 cannot be interpreted as a valid index of motor-related activity. The reason for this is illustrated in Figure 3 (top), where the ERP waveforms obtained in response to the laterally presented target letters at lateral occipital electrodes are displayed separately for sites ipsilateral and contralateral to the visual field of stimulation. There are systematic amplitude and latency differences between the ERP components obtained at ipsilateral and contralateral electrodes. The $\mathrm{P} 1$ component peaks about $20 \mathrm{msec}$ earlier over the contralateral occipital cortex, and the contralateral N1 amplitude is considerably larger than the N1 observed ipsilaterally. In addition, the contralateral N2 is also enhanced in comparison with the ipsilateral $\mathrm{N} 2$. These occipital asymmetries are partially due to the functional organization of the visual pathways, as information from the left and right hemifields is received by the contralateral occipital cortex. The latency difference between the contralateral and ipsilateral P1 component may reflect the time needed for visual information to travel through the corpus callosum in order to affect the ipsilateral hemisphere.
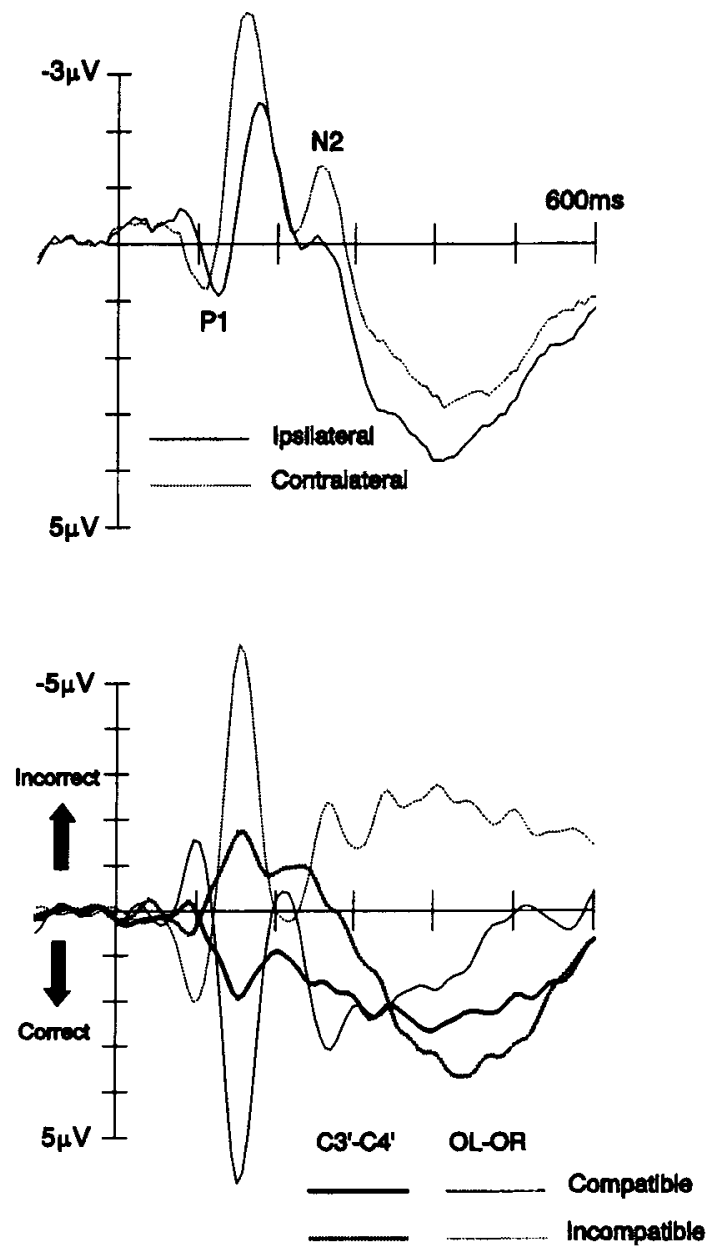

Figure 3. Top: Grand-averaged event-related brain potentials (ERPs) from 10 subjects, recorded at occipital electrodes. The lateral occipital recording sites were $O L$ and OR (located halfway between sites $\mathrm{O} 1$ and $\mathrm{T} 5$ and between $\mathrm{O} 2$ and $\mathrm{T} 6$, respectively, as specified by the $10-20$ system). The two ERP waveforms were obtained by averaging the ERPs recorded at OL and OR when the target letter was presented either ipsilateral (solid line) or contralateral (dashed line) to the respective electrode site. When compared with the ipsilateral electrode, the P1 component is elicited earlier, and the amplitudes of the $N 1$ and $N 2$ components are enlarged contralaterally. Bottom: LRP waveforms (thick lines) and difference waveforms obtained with the double subtraction method for lateral occipital sites $O L$ and $O R$ (thin lines), elicited in compatible trials (solid lines) and in incompatible trials (dashed lines). 
These sensory asymmetries over the occipital cortex are systematically confounded with the manipulation of spatial S-R compatibility. As can be seen from Figure 3 (top), an enlarged occipital N1 component is elicited contralateral to the side of the target. On compatible trials, target stimuli are presented ipsilateral to the required response, so that the enhanced negativity in the N1 time range at occipital electrodes is always located contralateral to the response. On incompatible trials, targets are presented contralateral to the required response, and the lateralized occipital negativity thus is ipsilateral to this response. These differential occipital activities elicited by laterally presented target stimuli are likely to be propagated by volume conduction and thus may be picked up by electrodes located over the motor cortex. If the ERPs recorded at $\mathrm{C}^{\prime}$ and $\mathrm{C}^{\prime}$ were systematically affected by these occipital asymmetries, the lateralization patterns observed over the lateral occipital cortex and over the motor cortex should be highly correlated.

This can be tested by comparing difference waveforms obtained with the double subtraction method for $C 3^{\prime}$ and $C 4^{\prime}$ (LRPs) with the corresponding difference waves obtained for OL and OR. Figure 3 (bottom) displays the double subtraction waves obtained for central electrodes (thick lines) and occipital electrodes (thin lines) separately for compatible and incompatible trials. The LRP deflections and the double subtraction waves derived from $\mathrm{OL}$ and $\mathrm{OR}$ are closely correlated during the first $200 \mathrm{msec}$ after stimulus onset. The first systematic deviation from baseline in the OL - OR difference waves around $100 \mathrm{msec}$ after stimulus onset reflects the latency difference of the $\mathrm{P} 1$ component between contralateral and ipsilateral occipital sites. This difference is mirrored by temporally corresponding, although rather small, LRP deviations. More importantly, the N1 amplitude difference between contralateral and ipsilateral occipital sites is strongly reflected in the $\mathrm{OL}$ - OR double subtraction waves as a large negativity for incompatible trials and a large positivity for compatible trials. The LRP waveforms recorded within this time interval show somewhat smaller, but closely parallel, effects (see ValleInclán, 1996, for a similar pattern of results).

The correlation of the LRP waveforms with the OL OR double subtraction waves shown in Figure 3 (bottom) strongly suggests that at least the initial part of the LRPs obtained in this experiment cannot be interpreted as an index of central motor activation. In the first $200 \mathrm{msec}$ after stimulus onset, the LRP also mirrors asymmetries between the ipsilateral and contralateral occipital cortex that are likely to be sensory in nature. The LRP reflects any enhanced negativity recorded over the motor cortex contralateral to the to-be-executed response, regardless of its origin. In situations in which horizontally lateralized stimuli are presented and response side is confounded with the side of presentation (as in manipulations of spatial S-R compatibility), it cannot be decided whether early LRP deflections are due to sensory asymmetries, motor-related asymmetries, or a combination of both. In these situations, the LRP cannot be used as an index of selective motor activation.

De Jong et al. (1994) employed a different procedure for measuring the automatic response activation processes that are due to spatial compatibility, one that is likely to overcome the problem of lateralized sensory asymmetries. They used stimuli and responses that were lateralized along the vertical axis and obtained LRP evidence for an early automatic activation of responses spatially corresponding to the stimulus location (see also Valle-Inclán, 1996). This procedure offers the additional advantage that the assignment of the left and right hand to upper and lower responses can be alternated between blocks. Such a manipulation is essential because stimuli located in the upper and lower visual field may not be equivalent with respect to the ERP activity generated at lateral posterior electrodes. Gunter, Wijers, Jackson, and Mulder (1994) recorded ERPs to single letters presented above and below a central fixation dot and found rather different ERP patterns, particularly at the midline occipital electrode $\mathrm{Oz}$. These differences may be due to the fact that upper-field stimuli are projected to the lower part of the primary visual cortex (the lower bank of the calcarine fissure), whereas stimuli presented below fixation are projected to the upper part. It is possible that this anatomical fact is also reflected in a differential ERP pattern elicited by upper-field and lower-field stimuli at lateral posterior electrodes. If the left and right hand were rigidly mapped to upper and lower response keys, measuring the LRP in response to vertically lateralized stimuli would also be subject to the problems discussed above.

\section{The Overlap of Motor and Nonmotor Activity in the LRP: The Case of Symmetrical Target/Nontarget Arrays}

The effects of spatial S-R compatibility on motor activation processes cannot be studied with the help of the LRP in experiments that employ horizontally lateralized single target stimuli because of the presence of sensory lateralizations that are confounded with the manipulation of compatibility. However, this problem may be overcome by using a modified S-R compatibility paradigm in which a lateralized target stimulus is presented simultaneously with a number of nontargets. If the resulting target/nontarget array is symmetrical, no sensory-evoked early asymmetries should be elicited, and the LRP might be interpreted as a valid index of motor activation.

This possibility was investigated in another experiment (Eimer, 1996, Experiment 1) in which target stimuli were presented in the left or right visual field together with three nontargets that were located in the contralateral visual field as well as above and below the central fixation cross. The horizontal and vertical distance of each stimulus from the screen center was $3.3^{\circ}$, which resulted in a symmetrical stimulus array. Blue and green squares served as targets, yellow squares as nontargets (see Figure 4, top), and all stimuli were approximately 


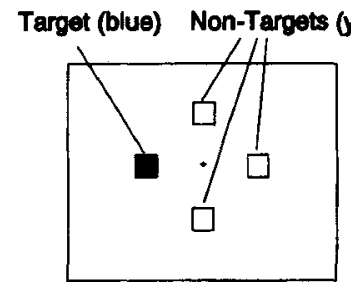

Left Response - Compatble

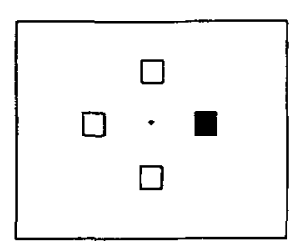

Left Response - Incompatible

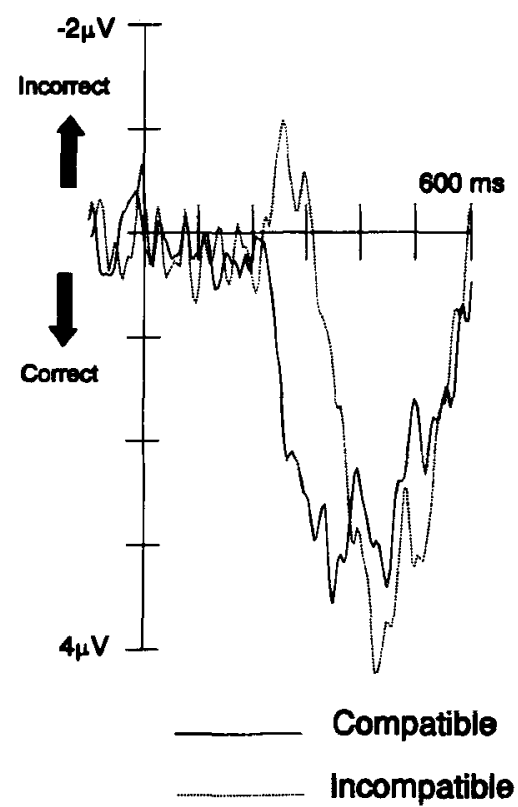

Figure 4. Top: Examples of stimulus displays in an experiment on spatial stimulus-response compatibility with symmetrical target/ nontarget arrays (Eimer, 1996, Experiment 1) in which target and response side could either be compatible (left side) or incompatible (right side). Bottom: Grand-averaged LRP waveforms from 10 subjects, elicited in compatible trials (solid line) and in incompatible trials (dashed line).

equiluminant. Ten subjects took part in this experiment. Half of them were instructed to respond with a left-hand response to blue squares and with a right-hand response to green squares, and this response assignment was reversed for the other 5 subjects. Target location (left or right visual field) was varied randomly. As before, a Simon effect was obtained in this experiment, with faster RTs in compatible than in incompatible trials ( $467 \mathrm{msec}$ vs. $489 \mathrm{msec}$ ). If this effect was due to an automatic activation of a response spatially corresponding to the location of the target, one should find evidence for an early activation of the correct response in compatible trials and of the incorrect response in incompatible trials. Figure 4 (bottom) shows the LRP waveforms obtained for compatible and incompatible trials. Between 230 and $300 \mathrm{msec}$ after stimulus onset, the LRP amplitude ob- tained for incompatible trials was negative (indicating an activation of the incorrect response), whereas it was positive on compatible trials.

A confounding of motor and sensory asymmetries was assumed to be eliminated in the present experiment because of the employment of symmetrical target/nontarget arrays. In the experiment reported in the previous section, sensory asymmetries were found to be maximal between 100 and $200 \mathrm{msec}$ after target onset, whereas Figure 4 shows that differential LRP effects started beyond $200 \mathrm{msec}$ in the present study. Figure 5 illustrates why these LRP waveforms can still not be regarded as valid indicators of motor activation. The ERP waveforms elicited at occipital electrodes (OL and OR) ipsilateral and contralateral to the visual field of target presenta-
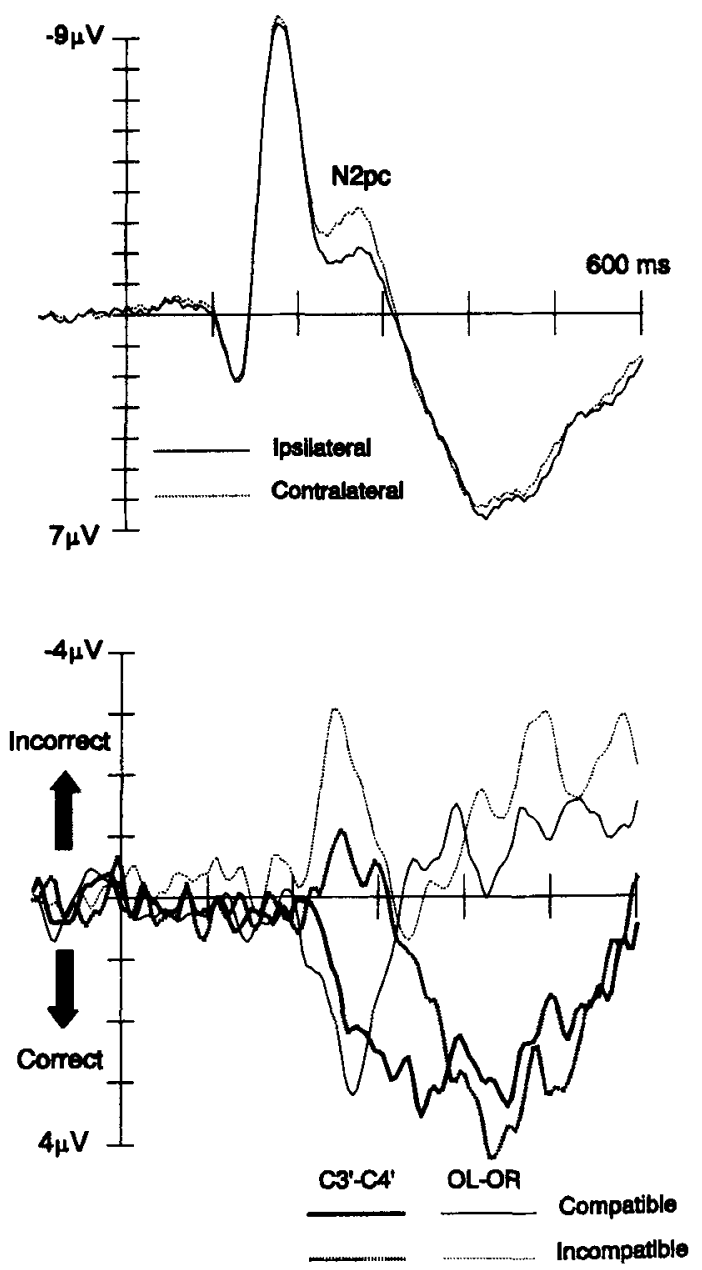

Figure 5. Top: Grand-averaged event-related brain potentials (ERPs) from 10 subjects, recorded at occipital electrodes (OL, $O R)$ ipsilateral (solid line) and contralateral (dashed line) to the side of the target. An N2pc component is elicited at contralateral sites. Bottom: LRP waveforms (thick lines) and difference waveforms obtained with the double subtraction method for lateral occipital sites $O L$ and $O R$ (thin lines), elicited in compatible trials (solid lines) and in incompatible trials (dashed lines). 
tion (Figure 5, top) reveal another systematic difference between ipsilateral and contralateral recording sites. An enlarged negativity was elicited in the N2 time range at electrodes contralateral to the visual field where the target stimulus was presented. This lateralized occipital component was first described by Luck and Hillyard (1994a, 1994b) and termed N2pc, since it is elicited at posterior electrodes contralateral to the location of a target stimulus. The N2pc has been interpreted as indicating the attentional selection of target stimuli, the selective inhibition of distractors, or both (Eimer, 1996; Luck \& Hillyard, 1994a, 1994b). Whatever the functional significance of this component might be, the fact that it is elicited by symmetrical target/nontarget arrays causes problems for the interpretation of the LRP that are analogous to the problems outlined above.

This is illustrated in Figure 5 (bottom), where the LRPs obtained in compatible and incompatible trials (thick lines) are compared with the respective double subtraction waves computed for lateral occipital sites (thin lines). The presence of the N2pc component is reflected by a negativity for incompatible trials and a positivity for compatible trials in the OL - OR difference waveforms between 200 and $320 \mathrm{msec}$ after target onset. Parallel, although somewhat smaller, deflections can be seen in the LRP waveforms within this time interval. The negative deflection found in the LRP for incompatible trials between 230 and $300 \mathrm{msec}$ after target onset closely parallels the negative-going deflection observed for the OL OR difference wave (see Wascher \& Wauschkuhn, 1996, for similar results). This suggests that the LRP deflections obtained in this experiment are at least in part a result of an asymmetric negativity generated at posterior sites that is propagated through volume conduction to $\mathrm{C3}^{\prime}$ and $\mathrm{C}^{\prime}$. Although the LRP effects may be partially due to motor-related activities, they are also influenced by processes responsible for the elicitation of the N2pc component (which are likely to be localized in the occipital cortex; see Luck \& Hillyard, 1994a). Again, the LRP cannot be unequivocally interpreted as an index for central response activation processes.

\section{Concluding Remarks}

The studies described in the previous two sections have shown that the computation of the LRP on the basis of the double subtraction method does not always guarantee that the resulting LRP waveforms are a pure measure of motor-related brain activity. In these experiments, additional lateralizations were elicited that were systematically confounded with the side of the to-be-executed response but were presumably caused by sensory asymmetries (elicited by left and right targets in singlestimulus displays) or by attentional processes (elicited by left and right targets in symmetrical target/nontarget arrays). The LRP deflections were temporally correlated with asymmetries obtained over occipital sites, and the occipital lateralizations were considerably larger in amplitude than were the corresponding LRP effects. This pattern of results makes it likely that these LRP deflections are, in large part, not generated in the motor cortex.

A way to determine whether LRP waveforms exclusively reflect motor-related activity is to record ERP waveforms not only from $\mathrm{C}^{\prime}$ and $\mathrm{C}^{\prime}$ but also from additional lateral electrode pairs at anterior or posterior sites or both, in order to perform separate double subtraction computations on these waveforms and to compare the resulting difference waves with the LRP (cf. Wascher \& Wauschkuhn, 1996, for an application of this procedure). When systematic deflections are obtained in these difference waves that are temporally correlated with the LRP effects and are at least of equal amplitude, there is reason to doubt that the LRP can be interpreted unequivocally. This procedure may not be only suitable for studies on spatial S-R compatibility but also for other experimental paradigms in which the LRP is used as the dependent variable and the presence of additional, presumably nonmotor, lateralizations cannot be excluded. Lateralized ERP activities may be elicited in response to asymmetrical stimuli (e.g., arrows) or stimulus arrays containing multiple stimuli (as, e.g., in studies on flanker compatibility effects or visual search); they also may be elicited prior to shifts of visual-spatial attention (see Yamaguchi, Tsuchiya, \& Kobayashi, 1994), during the preparation of saccadic eye movements (cf. Evdokimidis, Mergner, \& Lücking, 1992); and they may also be caused by reafferent information from the somatosensory cortex that follows the initiation of a movement (see Wauschkuhn, Wascher, \& Verleger, 1997). LRP waveforms should also be compared with double subtraction waves obtained from other scalp sites in situations where left-hand and right-hand responses are used to indicate the differential outcome of cognitive operations (e.g., present-absent judgments or word-nonword decisions), and these response mappings cannot simply be alternated between experimental blocks.

In summary, the LRP has been employed in numerous studies on mental chronometry because of its rather obvious functional relationship to response-related processes. To be interpretable as a valid index of selective central response activation, it has to be shown that it is not systematically affected by other ongoing brain activity. Under normal circumstances, the double subtraction method performed on the ERPs obtained at $\mathrm{C}^{\prime}$ ' and $\mathrm{C4}^{\prime}$ is well suited for this purpose. There are conditions, however, in which additional evidence from other recording sites is necessary in order to rule out the possibility that LRP effects are at least partially caused by processes unrelated to motor activation. When applied properly, the LRP may further serve as an important tool in the chronometric studies of human information processing.

\section{REFERENCES}

Arezzo, J., \& Vaughan, H. G. (1975). Cortical potentials associated with voluntary movements in the monkey. Brain Research, 88, 99-104. 
Böcker, K. B. E., Brunia, C. H. M., \& Cluitmans, P. J. M. (1994). A spatio-temporal dipole model of the readiness potential in humans: II. Foot movement. Electroencephalography \& Clinical Neurophysiology, 91, 286-294.

BRUNIA, C. H. M. (1980). What is wrong with the legs in motor preparation? Progress in Brain Research, 54, 232-236.

CoLEs, M. G. H. (1989). Modern mind-brain reading: Psychophysiology, physiology, and cognition. Psychophysiology, 26, 251-269.

Coles, M. G. H., Gratton, G., \& Fabiani, M. (1990). Event-related potentials. In J. T. Cacioppo \& L. G. Tassinary (Eds.), Principles of psychophysiology: Physical, social, and inferential elements (pp. 413455). New York: Cambridge University Press.

De Jong, R., Coles, M. G. H., Logan, G. D., \& Gratton, G. (1990). In search of the point of no return: The control of response processes. Journal of Experimental Psychology: Human Perception \& Performance, 16, 164-182.

De Jong, R., Liang, C. C., \& LaUber, E. (1994). Conditional and unconditional automaticity: A dual-process model of effects of spatial stimulus-response compatibility. Journal of Experimental Psychology: Human Perception \& Performance, 20, 731-750.

De Jong, R., Wierda, M., Mulder, G., \& Mulder, L. J. M. (1988). Use of partial stimulus information in response processing. Journal of Experimental Psychology: Human Perception \& Performance, 14, 682-692.

EIMER, M. (1993). Spatial cueing, sensory gating and selective response preparation: An ERP study on visuo-spatial orienting. Electroencephalography \& Clinical Neurophysiology, 88, 408-420.

EIMER, M. (1995). Stimulus-response compatibility and automatic response activation: Evidence from psychophysiological studies. Journal of Experimental Psychology: Human Perception \& Performance, 21, 837-854.

EIMER, M. (1996). The N2pc component as an indicator of attentional selectivity. Electroencephalography \& Clinical Neurophysiology, 99, 225-234.

Eimer, M., Goschke, T., Schlaghecken, F., \& Stürmer, B. (1996). Explicit and implicit learning of event sequences: Evidence from event-related brain potentials. Journal of Experimental Psychology: Learning, Memory, \& Cognition, 22, 1-18.

Eimer, M., Hommel, B., \& Prinz, W. (1995). S-R compatibility and response selection. Acta Psychologica, 90, 301-313.

Eviokimidis, I., Mergner, T., \& Lücking, C. H. (1992). Dependence of presaccadic cortical potentials on the type of saccadic eye movement. Electroencephalography \& Clinical Neurophysiology, 83, 179-191.

Gehring, W. J., Gratton, G., Coles, M. G. H., \& Donchin, E. (1992). Probability effects on stimulus evaluation and response processes. Journal of Experimental Psychology: Human Perception \& Performance, 18, 198-216.

Gemba, H., \& SASaKı, K. (1990). Potential related to no-go reaction in go/no-go hand movements with discrimination between tone stimuli of different frequencies in the monkey. Brain Research, 537, 340-344.

Gratton, G., Coles, M. G. H., Sirevaag, E. J., Eriksen, C. W., \& DonChIN, E. (1988). Pre- and poststimulus activation of response channels: A psychophysiological analysis. Journal of Experimental Psychology: Human Perception \& Performance, 14, 331-344.

Gratton, G., Kramer, A. F., Coles, M. G. H., \& Donchin, E. (1989) Simulation studies of latency measures of components of eventrelated brain potential. Psychophysiology, 26, 233-248.

Gunter, T. C., WiJers, A. A., JACKSON, J. L., \& Mulder, G. (1994). Visual spatial attention to stimuli presented on the vertical meridian: An ERP study. Psychophysiology, 31, 140-153.

HasbroucQ, T., \& GuiARD, Y. (1991). Stimulus-response compatibility and the Simon effect: Toward a conceptual clarification. Journal of Experimental Psychology: Human Perception \& Performance, 17, 246-266.

IKEDA, A., Lüders, H. O., Burgess, R. C., \& Shibasaki, H. (1992). Movement-related potentials recorded from supplementary motor area and primary motor area. Brain, 115, 1017-1043.

JASPER, H. H. (1958). The ten-twenty electrode system of the Interna- tional Federation. Electroencephalography \& Clinical Neurophysiology, 10, 371-375.

Kornblum, S., HasbroucQ, T., \& Osman, A. (1990). Dimensional overlap: Cognitive basis for stimulus-response compatibility-a model and taxonomy. Psychological Review, 97, 253-270.

KORNHUBER, H. H., \& DEECKE, L. (1965). Hirnpotentialänderungen bei Willkürbewegungen und passiven Bewegungen des Menschen: Bereitschaftspotential und reafferente Potentiale. Pflügers Archiv für die gesamte Physiologie, 248, 1-17.

KuTAs, M., \& Donchin, E. (1980). Preparation to respond as manifested by movement-related brain potentials. Brain Research, 202, 95-115.

Leuthold, H., Sommer, W., \& Ulrich, R. (1996). Partial advance information and response preparation: Inferences from the lateralized readiness potential. Journal of Experimental Psychology: General, 125, 307-323.

LuCK, S. J., \& Hillyard, S. A. (1994a). Electrophysiological correlates of feature analysis during visual search. Psychophysiology, 31, 291-308.

LuCK, S. J., \& Hiliyard, S. A. (1994b). Spatial filtering during visual search: Evidence from human electrophysiology. Journal of Experimental Psychology: Human Perception \& Performance, 20, 10001014.

Miller, J., \& HaCkley, S. A. (1992). Electrophysiological evidence for temporal overlap among contingent mental processes. Journal of Experimental Psychology: General, 121, 195-209.

Miller, J., PATterson, T., \& UlRich, R. (in press). A jackknife-based method for measuring LRP onset latency differences. Psychophysiology.

MILLER, J., \& ULRICH, R. (in press). Locus of the effect of the number of alternatives: Evidence from the lateralized readiness potential. Journal of Experimental Psychology: Human Perception \& Performance.

Oxada, Y. C., Williamson, S. J., \& Kaufman, L. (1982). Magnetic fields of the human sensorimotor cortex. International Journal of Neurophysiology, 17, 33-38.

Osman, A., Bashore, T. R., Coles, M. G. H., Donchin, E., \& Meyer, D. E. (1992). On the transmission of partial information: Inferences from movement-related brain potentials. Journal of Experimental Psychology: Human Perception \& Performance, 18, 217-232.

OSMAN, A., \& MOORE, C. M. (1993). The locus of dual-task interference: Psychological refractory effects on movement-related brain potentials. Journal of Experimental Psychology: Human Perception \& Performance, 19, 1292-1312.

POSNER, M. I. (1978). Chronometric explorations of mind. Hillsdale, NJ: Erlbaum.

REQUIN, J. (1985). Looking forward to moving soon: Ante factum selective processes in motor control. In M. I. Posner \& O. S. M. Marin (Eds.), Attention and Performance XI (pp. 147-167). Hillsdale, NJ: Erlbaum.

REQUIN, J., \& RIEHLE, A. (1995). Neural correlates of partial transmission of sensorimotor information in the cerebral cortex. Acta Psychologica, 90, 81-95.

RohrbauGH, J. W., \& GaillaRd, A. W. K. (1983). Sensory and motor aspects of the contingent negative variation. In A. W. K. Gaillard \& W. Ritter (Eds.), Tutorials in ERP research: Endogenous components (pp. 269-310). Amsterdam: North-Holland.

Schwarzenau, P., Falkenstein, M., Hoormann, J., \& Hohnsbein, J. (1998). A new method for the estimation of the onset of the lateralized readiness potential (LRP). Behavior Research Methods, Instruments, \& Computers, 30, 110-117.

SIMON, J. R. (1969). Reactions toward the source of stimulation. Journal of Experimental Psychology, 81, 174-176.

Smulders, F. T. Y., Kenemans, J. L., \& KoK, A. (1996). Effects of task variables on measures of the mean onset latency of LRP depend on the scoring method. Psychophysiology, 33, 194-205.

Smulders, F. T. Y., KoK, A., Kenemans, J. L., \& Bashore, T. R. (1995). The temporal selectivity of additive factor effects on the reaction process revealed in ERP component latencies. Acta Psychologica, 90, 97-109.

SOMmer, W., Leuthold, H., \& UlRICH, R. (1994). The lateralized 
readiness potential preceding brief isometric force pulses of different peak force and rate of force production. Psychophysiology, 31, 503-512.

SYNDULKo, K., \& LiNDSLEY, D. B. (1977). Motor and sensory determinants of cortical slow potential shifts in man. In J. E. Desmedt (Ed.), Attention, voluntary contraction and event-related cerebral potentials (pp. 97-131). Basel: Karger.

VALle-INCLÁN, F. (1996). The locus of interference in the Simon effect: An ERP study. Biological Psychology, 43, 147-162.

VAN Boxtel, G. J. M. (1994). Non-motor components of slow brain potentials. Unpublished doctoral dissertation, Katholieke Universiteit Brabant, Tilburg, the Netherlands.

VAN BoXTEL, G. J. M. (1998). Computational and statistical methods for analyzing event-related potential data. Behavior Research Methods, Instruments, \& Computers, 30, 87-102

VAughan, H., Costa, L. D., \& RitTeR, W. (1968). Topography of the human motor potential. Electroencephalography \& Clinical Neurophysiology, 25, 1-10.

WALLACE, R. J. (1971). S-R compatibility and the idea of a response code. Journal of Experimental Psychology, 88, 354-360.

Walter, W. G., Cooper, R., Aldridge, V. J., McCallum, W. C., \&
WINTER, A. L. (1964). Contingent negative variation: An electrical sign of sensorimotor association and expectancy in the human brain. Nature, 203, 380-384.

WASCHER, E., \& WAUSCHKUHN, B. (1996). The interaction of stimulusand response-related processes measured by event-related lateralizations of the EEG. Electroencephalography \& Clinical Neurophysiology, 99, 149-162.

WAUSCHKUHN, B., WASChER, E., \& VerLEGER, R. (1997). Lateralized cortical activity due to the preparation of saccades and finger movements: A comparative study. Electroencephalography \& Clinical Neurophysiology, 102, 114-124.

Yamaguchi, S., TsuchiYa, H., \& Kobayashi, S. (1994). Electroencephalographic activity associated with shifts of visuospatial attention. Brain, 117, 553-562.

\section{NOTE}

1. The experimental data referred to in this article were obtained from studies that have already been described elsewhere (Eimer, 1993 , 1996; Eimer et al., 1995). However, the LRP data presented here have not been previously published. 